\title{
InterSpot: Interactive Spammer Detection in Social Media
}

\author{
Kaize Ding, Jundong Li, Shivam Dhar, Shreyash Devan and Huan Liu \\ Arizona State University, Tempe, AZ 85281, USA \\ \{kaize.ding, jundong.li, sdhar3, srdevan, huan.liu\}@asu.edu
}

\begin{abstract}
Spammer detection in social media has recently received increasing attention due to the rocketing growth of user-generated data. Despite the empirical success of existing systems, spammers may continuously evolve over time to impersonate normal users while new types of spammers may also emerge to combat with the current detection system, leading to the fact that a built system will gradually lose its efficacy in spotting spammers. To address this issue, grounded on the contextual bandit model, we present a novel system for conducting interactive spammer detection. We demonstrate our system by showcasing the interactive learning process, which allows the detection model to keep optimizing its detection strategy through incorporating the feedback information from human experts.
\end{abstract}

\section{Introduction}

Social media services (e.g., Facebook, Youtube) have emerged as popular platforms for content sharing and information dissemination. The rapid growth of social media also provides malicious users a new and convenient medium to spread spamming contents for their noxious intentions. Those malicious users, also known as social spammers [Lee et al., 2010; Webb et al., 2008; Hu et al., 2014], are able to perform various attacks such as spreading fake news [Shu et al., 2017], disseminating phishing links [Hu et al., 2014], and promoting or even sabotaging the reputation of targeted products [Mukherjee et al., 2012]. The massive spamming contents generated by social spammers may have an adverse effect on the user experience on these social media platforms. Therefore, detecting social spammers is a vital research problem that has significant implications on keeping social media users from unwanted information that is generated by malicious attacks.

To counter these severe threats, extensive research efforts have been devoted to detecting social spammers with disruptive behaviors. Generally, a vast majority of existing methods can be classified into two categories. One family of methods are based on supervised learning techniques [Lee et al., 2010; Benevenuto et al., 2010; Hu et al., 2013]. For instance, [Benevenuto et al., 2010] proposed to adopt both content and behaviors of each user as its attributes and apply SVM to train a social spammer classifier. [Lee et al., 2010] proposed to deploy honeypots in social networks for collecting training data, and learn a spam detector using extracted feature vectors. The training of spammer detection classifiers heavily relies on the assumption that a sufficient amount of labeled samples are available. Nevertheless, due to the prohibitive cost for accessing the ground truth social spammers, it is unrealistic to collect a large amount of annotated data. As alternative solutions, unsupervised methods [Uemura et al., 2008; Bouguessa, 2011; Tan et al., 2013; Ding et al., 2019a] have received a surge of research interests and achieved extensive success by characterizing the difference between legitimate users and social spammers. However, as spammers can quickly evolve and new types of spammers may also arise, a trained model will lose its power owing to the inability of capturing such environment changes. Fortunately, advanced research in human-in-theloop machine learning [Holzinger, 2016; Huang et al., 2018; Ding et al., 2019b] show that by interactively incorporating expert knowledge in the learning process, the model is able to sense the environment changes and the performance can be remarkably improved. As such, there is an urgent need for developing a system that supports us to spot spammers in social media in an interactive fashion.

Contribution. In this study, on the basis of the contextual multi-armed bandit algorithm, we present a novel system: InterSpot $^{1}$, which facilitates the detection of social spammer in an interactive manner. By continuously incorporating the expert knowledge about social spammers into the learning process, our system is able to constantly optimize the detection strategy for tracing the environment changes and thus achieve superior detection performance in practical usage.

\section{System Overview}

In this section, we carefully illustrate the overview of our proposed system on three aspects: (1) studied dataset; (2) the proposed algorithm; and (3) system interface.

\subsection{Studied Dataset}

We showcase our system on a real-world spammer detection dataset: YelpCHI. This dataset is collected from Yelp.com and

\footnotetext{
${ }^{1}$ A demo video can be found at https://youtu.be/oW1pOD6zc1g
} 


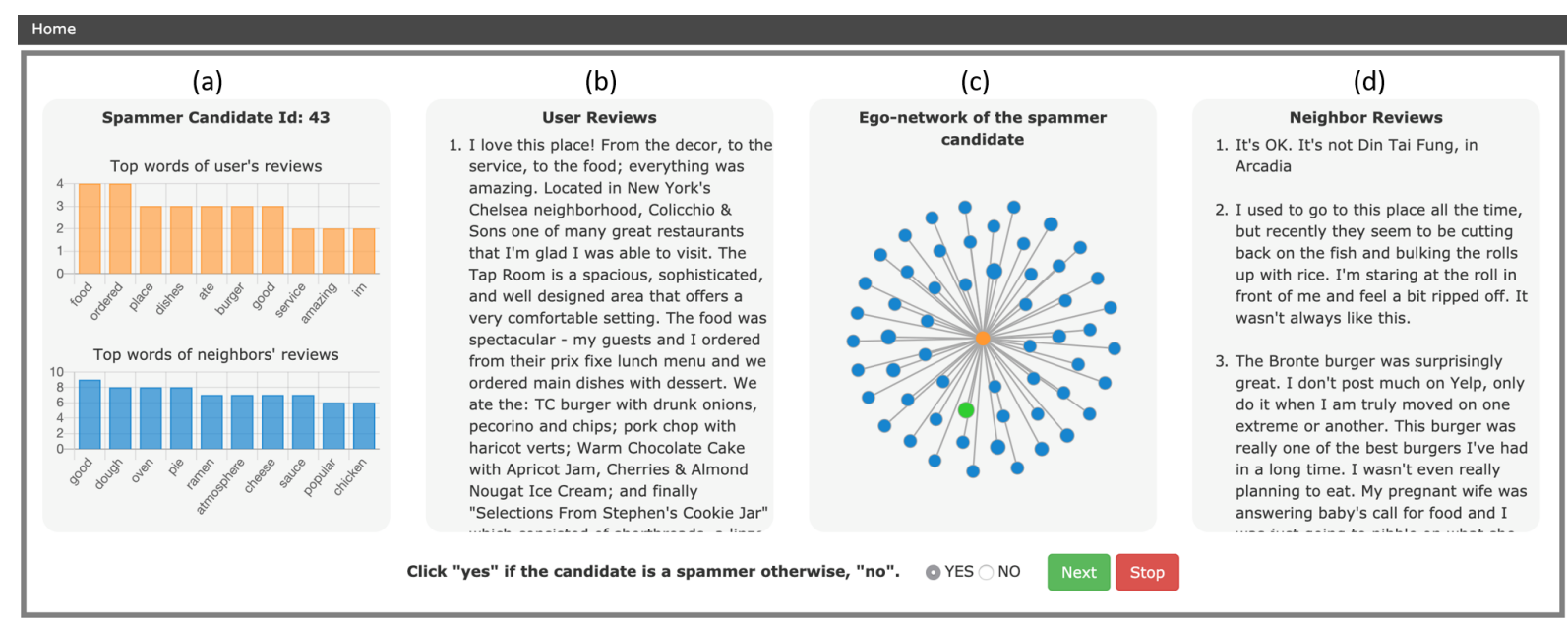

Figure 1: An illustrative example of the interactive social spammer detection framework (InterSpot).

has been widely used in previous research [Mukherjee et al., 2013; Rayana and Akoglu, 2015]. The dataset includes reviews by 38,063 reviewers on 201 different hotels and restaurants. According to the results from Yelp anti-fraud filter, we are able to divide the reviewers into two classes: authors of fake reviews (social spammers), and authors of real reviews (legitimate users). We create a reviewer-reviewer network following the way of [Kaghazgaran et al., 2018]. Additionally, we apply the bag-of-words model on the whole reviews to extract the feature vector of each user for model learning.

\subsection{Algorithm Description}

Our system possesses a contextual multi-armed bandit (CMAB) backbone which attempts to address the problem of spammer detection in an interactive manner. In many real-world applications (e.g., recommender systems [Li et al., 2010a; Bouneffouf et al., 2012] and display advertising [Li et al., 2010b; Chapelle and Li, 2011]), we often need to tackle the so-called exploration-exploitation dilemma where it is important to make a trade-off between exploiting the current accumulated knowledge and exploring new knowledge by trying out the unknown space. In our scenario, we also need to address the dilemma between exploiting existing known types of spammers and exploring new types of spammers, to achieve superior detection performance. Therefore, contextual multi-armed bandit algorithm [Chu et al., 2011; Li et al., 2010a; Lu et al., 2010] is a principled tool that we can resort to for conducting interactive learning.

To formulate our social spammer detection problem within the $K$-armed contextual bandit framework, we first partition the $N$ users into $K$ different clusters. The reason is that the users in one cluster can be considered as samples drawn from the distribution behind a bandit arm. Thus for each user, we can regard the cluster it belongs to as an arm to pull, and when we pull that particular arm, we consider its features as the contextual feature vector. With the contextual feature vectors of all the users $\left\{\mathbf{x}_{i}\right\}_{i=1}^{i=N}$, at each trail $t \in\{1, \ldots, T\}$, our system selects one suspicious user $i_{t}$ and queries the human expert if it is considered as social spammer or not. In order to model both user features and network structure information, follow the framework of LinUCB [Li et al., 2010a], the expected payoff of selecting user $i$ can be defined as:

$$
\begin{aligned}
& r_{i}=\mathbf{x}_{i}^{\top} \boldsymbol{\theta}_{a(i)}+\alpha \mathbf{y}_{i}^{\top} \boldsymbol{\phi}_{a(i)} \\
& \text { s.t. } \mathbf{y}_{i}=\operatorname{AGGR}\left(\left\{\mathbf{x}_{j}, \forall j \in \mathcal{N}(i)\right\}\right) \text {, }
\end{aligned}
$$

where $\alpha$ is a controlling parameter to balance the impact between two information modalities and $a(i)$ represents the arm that user $i$ belongs to. $\boldsymbol{\theta}$ and $\phi$ are the coefficient vectors for modeling user features and network structure, respectively. $\operatorname{AGGR}()$ is a predefined aggregator function which aggregates the features from neighbors, and one prevalent choice is to use the mean operator [Xu et al., 2018]. Once the human expert provides his feedback information, our system will incorporate the feedback and can update its selection strategy according to the observed reward $r_{i_{t}} \in\{0,1\}$. We repeat the whole process until we run out of the $T$ queries budget.

\subsection{System Demonstration}

As visually depicted in Figure 1, once the input dataset is uploaded, the system will enter into the interactive detection process. In each round, the system will present one candidate spammer along with its auxiliary information to the human expert. To facilitate the human expert to assess the abnormality of each candidate, in our demo system, we provide four classes of auxiliary information. Part (a) shows some statistics (e.g., top words) about the reviews of the spammer candidate (up) and its neighbors (down). Part (b) shows the original reviews of the spammer candidate. Additionally, the ego-network of the spammer candidate is shown in Part (c). When the human expert clicks one of the neighboring nodes, the original reviews of this neighbor will be displayed in Part (d). Note that other auxiliary information can also be exploited for further extension. After the human expert clicks the button according to his domain knowledge, the feedback information will be integrated back into the spammer detection model to update its selection strategy at the next iteration. This interactive process will iterate until the human expert stops the algorithm or the budget is used up. 


\section{References}

[Benevenuto et al., 2010] Fabricio Benevenuto, Gabriel Magno, Tiago Rodrigues, and Virgilio Almeida. Detecting spammers on twitter. In Collaboration, electronic messaging, anti-abuse and spam conference (CEAS), volume 6, page 12, 2010.

[Bouguessa, 2011] Mohamed Bouguessa. An unsupervised approach for identifying spammers in social networks. In 2011 IEEE 23rd International Conference on Tools with Artificial Intelligence, pages 832-840, 2011.

[Bouneffouf et al., 2012] Djallel Bouneffouf, Amel Bouzeghoub, and Alda Lopes Gançarski. A contextualbandit algorithm for mobile context-aware recommender system. In International Conference on Neural Information Processing, pages 324-331, 2012.

[Chapelle and Li, 2011] Olivier Chapelle and Lihong Li. An empirical evaluation of thompson sampling. In Advances in neural information processing systems, pages 22492257, 2011.

[Chu et al., 2011] Wei Chu, Lihong Li, Lev Reyzin, and Robert Schapire. Contextual bandits with linear payoff functions. In Proceedings of the 14th International Conference on Artificial Intelligence and Statistics, pages 208214, 2011.

[Ding et al., 2019a] Kaize Ding, Jundong Li, Rohit Bhanushali, and Huan Liu. Deep anomaly detection on attributed networks. In SIAM International Conference on Data Mining (SDM), 2019.

[Ding et al., 2019b] Kaize Ding, Jundong Li, and Huan Liu. Interactive anomaly detection on attributed networks. In Proceedings of the 12th ACM International Conference on Web Search and Data Mining, pages 357-365, 2019.

[Holzinger, 2016] Andreas Holzinger. Interactive machine learning for health informatics: when do we need the human-in-the-loop? Brain Informatics, 3(2):119-131, 2016.

[Hu et al., 2013] Xia Hu, Jiliang Tang, Yanchao Zhang, and Huan Liu. Social spammer detection in microblogging. In Proceedings of the 23rd International Joint Conference on Artificial Intelligence, 2013.

[Hu et al., 2014] Xia Hu, Jiliang Tang, and Huan Liu. Online social spammer detection. In Twenty-Eighth AAAI Conference on Artificial Intelligence, 2014.

[Huang et al., 2018] Xiao Huang, Qingquan Song, Jundong $\mathrm{Li}$, and Xia Hu. Exploring expert cognition for attributed network embedding. In Proceedings of the 11th ACM International Conference on Web Search and Data Mining, pages 270-278, 2018.

[Kaghazgaran et al., 2018] Parisa Kaghazgaran, James Caverlee, and Anna Squicciarini. Combating crowdsourced review manipulators: A neighborhood-based approach. In Proceedings of the 11th ACM International Conference on Web Search and Data Mining, pages 306-314, 2018.
[Lee et al., 2010] Kyumin Lee, James Caverlee, and Steve Webb. Uncovering social spammers: social honeypots+ machine learning. In Proceedings of the 33rd international ACM SIGIR conference on Research and development in information retrieval, pages 435-442, 2010.

[Li et al., 2010a] Lihong Li, Wei Chu, John Langford, and Robert E Schapire. A contextual-bandit approach to personalized news article recommendation. In Proceedings of the 19th international conference on World wide web, pages 661-670, 2010.

[Li et al., 2010b] Wei Li, Xuerui Wang, Ruofei Zhang, Ying Cui, Jianchang Mao, and Rong Jin. Exploitation and exploration in a performance based contextual advertising system. In Proceedings of the 16th ACM SIGKDD international conference on Knowledge discovery and data mining, pages 27-36, 2010.

[Lu et al., 2010] Tyler Lu, Dávid Pál, and Martin Pál. Contextual multi-armed bandits. In Proceedings of the 13th international conference on Artificial Intelligence and Statistics, pages 485-492, 2010.

[Mukherjee et al., 2012] Arjun Mukherjee, Bing Liu, and Natalie Glance. Spotting fake reviewer groups in consumer reviews. In Proceedings of the 21st international conference on World Wide Web, pages 191-200. ACM, 2012.

[Mukherjee et al., 2013] Arjun Mukherjee, Vivek Venkataraman, Bing Liu, and Natalie Glance. What yelp fake review filter might be doing? In Proceedings of 7 th international AAAI conference on weblogs and social media, 2013.

[Rayana and Akoglu, 2015] Shebuti Rayana and Leman Akoglu. Collective opinion spam detection: Bridging review networks and metadata. In Proceedings of the 21th acm sigkdd international conference on knowledge discovery and data mining, pages 985-994, 2015.

[Shu et al., 2017] Kai Shu, Amy Sliva, Suhang Wang, Jiliang Tang, and Huan Liu. Fake news detection on social media: A data mining perspective. ACM SIGKDD Explorations Newsletter, 19(1):22-36, 2017.

[Tan et al., 2013] Enhua Tan, Lei Guo, Songqing Chen, Xiaodong Zhang, and Yihong Zhao. Unik: Unsupervised social network spam detection. In Proceedings of the 22nd ACM international conference on Information \& Knowledge Management, pages 479-488, 2013.

[Uemura et al., 2008] Takashi Uemura, Daisuke Ikeda, and Hiroki Arimura. Unsupervised spam detection by document complexity estimation. In International Conference on Discovery Science, pages 319-331, 2008.

[Webb et al., 2008] Steve Webb, James Caverlee, and Calton $\mathrm{Pu}$. Social honeypots: Making friends with a spammer near you. In $C E A S$, pages 1-10, 2008.

[Xu et al., 2018] Keyulu Xu, Weihua Hu, Jure Leskovec, and Stefanie Jegelka. How powerful are graph neural networks? arXiv preprint arXiv:1810.00826, 2018. 